\title{
ANALYTIC CONTINUATION, ENVELOPES OF HOLOMORPHY, AND PROJECTIVE AND DIRECT LIMIT SPACES
}

\author{
BY
}

\section{ROBERT CARMIGNANI}

\begin{abstract}
For a Riemann domain $\Omega$, a connected complex manifold where $\boldsymbol{n}(\boldsymbol{n}=$ dimension) globally defined functions form a local system of coordinates at every point, and an arbitrary holomorphic function $f$ in $\Omega$, the "Riemann surface" $\Omega_{f}$, a maximal holomorphic extension Riemann domain for $f$, is formed from the direct limit of a sequence of Riemann domains. Projective limits are used to construct an envelope of holomorphy for $\Omega$, a maximal holomorphic extension Riemann domain for all holomorphic functions in $\Omega$, which is shown to be the projective limit space of the "Riemann surfaces" $\Omega_{f}$. Then it is shown that the generalized notion of envelope of holomorphy of an arbitrary subset of a Riemann domain can also be characterized in a natural way as the projective limit space of a family of "Riemann surfaces".
\end{abstract}

Introduction. Unlike the case in $\mathbf{C}^{\mathbf{1}}$, it was observed by Hartogs [10] that there are special domains in $\mathbf{C}^{n}$ such that all holomorphic functions in such a special domain can be holomorphically extended to a larger domain. Thullen in [16] introduced the notion of "Regularitätshulle" of a "bereich" (domain) and refuted a conjecture of Almer by giving an example of a Hartogs domain in $\mathbf{C}^{2}$ such that every holomorphic function could be holomorphically continued, but not always in a univalent manner, to a larger domain in $\mathbf{C}^{2}$. Later that year Cartan and Thullen in [6] defined the "Regularitätshülle" of a "domain" to be the "durchschnitt" of the family of "domains of holomorphy" of functions holomorphic in the given "domain". The idea of "durchschnitt" was generalized in [1] (see also [3, p. 179]) and is similar to the notion of a projective (inverse) limit space. The "durchschnitt" of a family of sets is a subset of the projective limit of this family, but its topology is stronger than the induced topology on it from the projective limit space. Given a family of domains in the plane with a point in common, the "durchschnitt" of this family is the interior of the intersection, while the projective limit space is homeomorphic to the intersection.

Received by the editors November 30, 1973 and, in revised form, April 19, 1974.

AMS (MOS) subject classifications (1970). Primary 32D10, 32D05; Secondary 54B25, 32E99, $52 \mathrm{~A} 20$.

Key words and phrases. Riemann domain, direct limit space, domain of holomorphy, projective limit, projective limit spaces, envelope of holomorphy, Stein manifold, holomorphically convex sets, convex hull. 
If the "Regularitätshülle" of a "domain" contained an isomorphic image of the given "domain", this would correspond to requiring that the map $\chi$ in Definition 2.1 be injective.

Cartan [7], by means of the sheaf of germs of holomorphic functions on $\mathbf{C}^{n}$ (whose topology corresponds to the topology of the "durchschnitt"), proved the existence of an envelope of holomorphy for an arbitrary Riemann domain (compare [13]). Cartan proved that it was pseudoconvex (based on the results in [6]) and that it was unique up to biholomorphism. Oka's solution of the Levi problem in [14] established that the envelope of holomorphy is a Stein manifold. In [15] Rossi constructed an envelope of holomorphy of a Riemann domain $\Omega$, which is characterized by the spectrum of the algebra of holomorphic functions in $\Omega$. Bishop in [2] , using Rossi's characterization, proved the envelope of holomorphy is Stein without relying on Oka's pseudoconvexity theorem.

In this paper we characterize the envelope of holomorphy of a Riemann domain $\Omega$ as the projective limit space of the family of "Riemann surfaces" $\Omega_{f}$ (domain of holomorphy of $f$ ) of all holomorphic functions $f$ in $\Omega$. We do not require that $O(\Omega)$ separate points in $\Omega$ and each $\Omega_{f}$ is characterized as the direct limit space of a sequence of Riemann domains.

First we prove (Lemma 1.4) that a sequence of Riemann domains with certain properties defines a direct spectrum (as defined in [8, p. 420], also called direct system) whose direct (inductive) limit space is a Riemann domain with the same properties. By forming power series expansions and by repeated applications of 1.4 we construct the "Riemann surface" $\Omega_{f}$ of an arbitrary holomorphic function $f$ in $\Omega$, which is the "domain of holomorphy" for $f$ in the sense of [9]. Although $O(\Omega)$ need not separate points in $\Omega$, we prove that for each $f$ there is a subset of $O\left(\Omega_{f}\right)$ which separates points in $\Omega_{f}$.

In $\S 2$ we construct an envelope of holomorphy for any Riemann domain $\Omega$, where $O(\Omega)$ need not separate points. We do this by preordering $O(\Omega)$ and then considering $E^{\prime}(\Omega)$, a special subset of the projective limit of the sets $\Omega_{f}$, which is given the subspace topology of $\left(\Pi \Omega_{f}, S\right)$ where $S$ is a certain stronger topology than the cartesian product space topology. $E^{\prime}(\Omega)$ corresponds to the classical notion of "durchschnitt" of the "bereichen" $\Omega_{f}$ mentioned earlier. We prove that $E^{\prime}(\Omega)$ is an envelope of holomorphy for $\Omega$ (Theorem 2.15) using the lemmas that precede 2.15 . That $E^{\prime}(\Omega)$ is Stein follows from a theorem due to Bishop. Lemmas 2.17 and 2.18 together with some well-known facts about projective limit spaces establish that the projective limit space $\widetilde{\Omega}$ of the projective spectrum (system) $\left\{\Omega_{f} ; \Psi_{f g}\right\}$ over $O(\Omega)$ is an envelope of holomorphy for $\Omega$ 
and that a certain subset of $O(\widetilde{\Omega})$ separates points in $\widetilde{\Omega}$. Also, we obtain that $\widetilde{\Omega}=E^{\prime}(\Omega)$ (Corollary 2.20).

For an example of a Riemann domain $\Omega$ for which $O(\Omega)$ does not separate points, but whose envelope of holomorphy is a polydisc, see [3, p. 177].

In $\S 3$ we first give two easy theorems for envelopes of holomorphy of Riemann domains which follow immediately from the projective limit space characterization.

Harvey and Wells introduced in [11] the notion of an envelope of holomorphy of an arbitrary subset $S$ of a Stein manifold which is defined to be the projective limit space of the Rossi envelopes of holomorphy of a fundamental system of neighborhoods of $S$. Theorem 3.3 shows that the Harvey-Wells envelope of holomorphy of an arbitrary connected set $S$ in a Riemann domain can be characterized as the projective limit space of the "Riemann surfaces" $\Omega_{f}$, where $f \in O(S)$. This is then used to obtain a characterization of holomorphically convex connected sets in a holomorphically convex Riemann domain, which is given by Theorem 3.4 .

Finally in a remark we show that the notion of envelope of holomorphy of a Riemann domain viewed as the projective limit space of "Riemann surfaces" is a formal analogue of the notion of convex hull of a domain.

It would be interesting to see if this projective limit space characterization of a Riemann domain is helpful in quantum field theory since the applications to this area of physics led Vladimirov to write in [17, p. VII], "methods of constructing envelopes of holomorphy ... are of supreme interest in quantum field theory."

The author would like to thank Professor F. Reese Harvey and the referee for several helpful suggestions.

I. Notation and preliminaries. $\alpha$ denotes an $n$-tuple $\left(\alpha_{1}, \ldots, \alpha_{n}\right)$ where $\alpha_{j}$ is a nonnegative integer, $1 \leqslant j \leqslant n$. We shall call $\alpha$ a multiorder. $|\alpha|=$ $\alpha_{1}+\cdots+\alpha_{n}$ and $\alpha !=\alpha_{1} ! \cdots \alpha_{n} !, \partial^{\alpha}=\left(\partial / \partial z_{1}\right)^{\alpha_{1}} \cdots\left(\partial / \partial z_{n}\right)^{\alpha_{n}}$ and $(z-\eta)^{\alpha}=$ $\left(z_{1}-\eta_{1}\right)^{\alpha_{1}} \cdots\left(z_{n}-\eta_{n}\right)^{\alpha_{n}} . A \backslash B$ is a notation for $A \cap \mathbf{C} B$ where $\mathbf{C} B$ denotes the complement of $B$. $A \subset \subset B$ means $\bar{A}$ is a compact subset of $B$. $f \mid A$ means the restriction of $f$ to $A$ and $\partial A$ is the boundary of $A$. X/R means the quotient space of $X$ determined by the equivalence relation $R . \Delta(z, \epsilon)$ denotes the poly. $\operatorname{disc}\left\{w \in \mathbf{C}^{n}:\left|w_{j}-z_{j}\right|<\epsilon, 1 \leqslant j \leqslant n\right\}$.

We take the following definition of Riemann domain:

Definition 1.1. A complex connected manifold $\Omega$ of dimension $n$ is called a Riemann domain if there is a local biholomorphism $\pi: \Omega \rightarrow \mathrm{C}^{n}$, that is, $\pi$ forms a local system of coordinates at every point ( $\pi$ is called the spread of $\Omega$ in $\mathbf{C}^{n}$ ). 
For $z \in \Omega$, we denote by $N(z, \epsilon)$ the neighborhood of $z$ which is mapped biholomorphically by $\pi$ onto $\Delta(\pi(z), \epsilon)$. The distance function on $\Omega$ is defined by

$$
d(z)=\sup \{\epsilon: N(z, \epsilon) \text { exists }\} \text {. }
$$

A function $f$ is holomorphic on the Riemann domain $\Omega$ if for each $z \in \Omega$, $f \circ \psi_{z}$ is holomorphic on $\Delta(\pi(z), \epsilon)$ for all $\epsilon<d(z)$ where $\psi_{z}$ is the inverse of the restriction of $\pi$ to $N(z, \epsilon)$. The space of holomorphic functions on $\Omega$ is denoted by $O(\Omega)$. Any subset $A$ of $O(\Omega)$ is said to separate points in $\Omega$ if whenever $z_{1}$ and $z_{2}$ are different points in $\Omega$, then $f\left(z_{1}\right) \neq f\left(z_{2}\right)$ for some $f \in A$. It is often required in the definition of Riemann domain that holomorphic functions separate points, however, we do not need to do so in this work.

Let $f \in O(\Omega)$, we define

$\tau\left(f, z_{1}\right)=\sup \left\{\epsilon:\right.$ the power series for $f \circ \psi_{z_{1}}$ converges in $\left.\Delta\left(\pi\left(z_{1}\right), \epsilon\right)\right\}$. It follows from the study of Reinhardt domains that $\pi\left(f, z_{1}\right) \geqslant d\left(z_{1}\right)$.

The proof of Proposition 2 of $[9$, p. 44] shows that a Riemann domain $\Omega$ is countable at infinity, that is, $\Omega$ is a countable union of compact sets.

Let $K$ be a compact subset of $\Omega$; we define the $O(\Omega)$-hull $\hat{K}_{\Omega}$ of $K$ by

$$
\hat{K}_{\Omega}=\{z \in \Omega:|f(z)| \leqslant \sup |f|, f \in O(\Omega)\} .
$$

$\Omega$ is said to be holomorphically convex if $\hat{K}_{\Omega} \subset \subset \Omega$ for every compact set $K \subset$ $\Omega$.

Definition 1.2. A pair $(\Omega, f)$ is a Riemann domain $\Omega$ together with a function $f \in O(\Omega)$.

Definition 1.3. The pair $(\widetilde{\Omega}, \widetilde{f})$ is called a holomorphic extension of the pair $(\Omega, f)$ if there is a local biholomorphism $\chi: \Omega \rightarrow \widetilde{\Omega}$ such that $\widetilde{f} \circ \chi=f$ in $\Omega$ and $\tilde{\pi} \circ \chi=\pi$ in $\Omega$ (where $\pi$ and $\tilde{\pi}$ are the spreads of $\Omega$ and $\widetilde{\Omega}$ respectively). If $\chi$ is also injective, then $(\widetilde{\Omega}, \widetilde{f})$ is called a natural holomorphic extension of $(\Omega, f)$.

REMARK. Let $\pi_{j}$ denote the $j$ th coordinate of $\pi$. Then the condition $\tilde{\pi}$ 。 $\chi=\pi$ in 1.3 means that the $j$ th coordinate of $\pi$ extends to the $j$ th coordinate of

We now construct the "Riemann surface" or maximal holomorphic extension of a pair $(\Omega, f)$ by constructing a sequence of Riemann domains $\left\{\Omega_{n}\right\}$, where each $\Omega_{n}$ is the direct limit (inductive limit) of a sequence of Riemann domains, and then taking the direct limit of $\left\{\Omega_{n}\right\}$. We give this dressed up version of the classical construction of the "Riemann surface" $\Omega_{f}$ for $(\Omega, f)$ rather than invoking the more elegant sheaf theoretic result because of duality between direct limits and projective limits (inverse limits) and because we obtain that a special proper subset of $O\left(\Omega_{f}\right)$ separates points in $\Omega_{f}$, and that if $\Omega \subset C^{n}$, then $\Omega$ imbeds in $\Omega_{f}$. 
We shall repeatedly apply

LEMMA 1.4. Let $\left\{\left(\Omega_{i}, f_{i}\right): i=1,2, \ldots\right\}$ be a sequence of pairs such that for each $i,\left(\Omega_{i+1}, f_{i+1}\right)$ is a holomorphic extension of $\left(\Omega_{i}, f_{i}\right)$ and $\left\{\partial^{\alpha} f_{i}: \alpha\right.$ is any multiorder $\} \cup\left\{\pi_{i_{j}} ; j=1, \ldots, n\right\}$ (where $\pi_{i j}$ is the $j$ th coordinate of the spread $\pi_{i}: \Omega_{i} \rightarrow \mathrm{C}^{n}$ ) separates points in $\Omega_{i}$. Then whenever $i \leqslant j$, there exists a local biholomorphism $\psi_{i j}: \Omega_{i} \rightarrow \Omega_{j}$ such that the family $\left\{\Omega_{i} ; \psi_{i j}\right\}$ is a direct spectrum and its direct limit space $\Omega^{\infty}$ can be given the structure of a Riemann domain with spread $\pi^{\infty}$. Furthermore, there exists $f^{\infty} \in O\left(\Omega^{\infty}\right)$ such that $\left(\Omega^{\infty}, f^{\infty}\right)$ is a holomorphic extension of $\left(\Omega_{i}, f_{i}\right)$ for each $i$ and $\left\{\partial^{\alpha} f^{\infty}: \alpha\right.$ is any multiorder $\} \cup\left\{\pi_{j}^{\infty}: j=1, \ldots, n\right\}$ (where $\pi_{j}^{\infty}$ is the $j$ th coordinate of $\pi^{\infty}$ ) separates points in $\Omega^{\infty}$.

Proof. We have the commutative diagram:

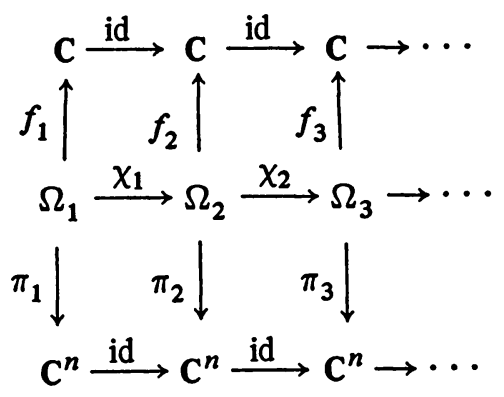

For $i \leqslant j$, we define $\psi_{i j}: \Omega_{i} \rightarrow \Omega_{j}$ by $\psi_{i j}=\chi_{j} \circ \cdots \circ \chi_{i}$. Then $\left\{\Omega_{i} ; \psi_{i j}\right\}$ is a direct spectrum and the families $\left\{\pi_{i}: i=1,2, \ldots\right\}$ and $\left\{f_{i}: i=1,2, \ldots\right\}$ are maps of this direct spectrum into $\mathbf{C}^{n}$ and $\mathbf{C}$ respectively. Let $\rho_{i}$ be the composition of the projection $\rho: \Sigma \Omega_{i} \rightarrow \Omega^{\infty}$ and the inclusion map $\iota_{i}: \Omega_{i} \rightarrow \Sigma \Omega_{i}$ (where $\Sigma \Omega_{i}$ is the free union of the spaces $\Omega_{i}$ and $\Omega^{\infty}=\Sigma \Omega_{i} / R$ where $R$ is the equivalence relation in $\Sigma \Omega_{i}$ defined by $z R w$ if there are points $z_{i} \in \Omega_{i}$ and $w_{j} \in \Omega_{j}$ with $\iota_{i}\left(z_{i}\right)=z$ and $\iota_{j}\left(w_{j}\right)=w$, such that for some integer $k, i \leqslant k \geqslant j$, $\left.\psi_{i k}\left(z_{i}\right)=\psi_{j k}\left(w_{j}\right)\right)$. By 1.5 of $\left[8\right.$, p. 422], there exist continuous maps $\pi^{\infty}$ and $f^{\infty}$ such that for each $i$, the diagram

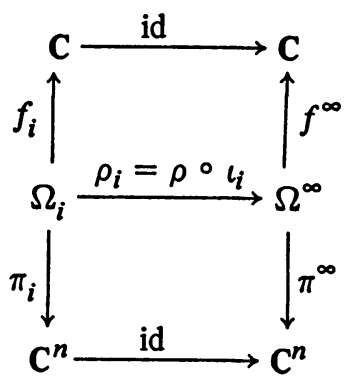

is commutative. We see that each $\rho_{i}$ is locally injective since each $\pi_{i}$ is locally 
injective. We now use this fact to show that $\rho_{i}$ is a local homeomorphism. Let $U$ be any open set in $\Sigma \Omega_{i}$. Take any $z \in \rho^{-1}(\rho(U))$ and find $u \in U$ such that $\rho(z)=\rho(u)$. By the definition of $\Omega^{\infty}$ there exist integers, $i, j, k$ and points $z_{i} \in$ $\Omega_{i}$ and $u_{j} \in \Omega_{j}$ such that $\psi_{i k}\left(z_{\imath}\right)=\psi_{j k}\left(u_{j}\right)$ where $\iota_{i}\left(z_{i}\right)=z$ and $\iota_{j}\left(u_{j}\right)=u$. Since $\psi_{i k}$ and $\psi_{j k}$ are local biholomorphisms we can find neighborhoods $N_{i}$ of $z_{i}$ and $M_{j}$ of $u_{j}$ such that $\psi_{i k}\left(N_{i}\right)=\psi_{j k}\left(M_{j}\right)$ where $\iota_{j}\left(M_{j}\right)=M \subset U$. Hence there is a neighborhood $N$ of $z$, where $N=\iota_{i}\left(N_{i}\right)$, such that $\rho(N) \subset \rho(U)$. This proves that $\rho^{-1}(\rho(U))$ is open in $\Sigma \Omega_{i}$. Since $\rho$ is an identification, $\rho(U)$ must be open in $\Omega^{\infty}$. Thus $\rho$ is an open map, and so $\rho_{i}$, being the composition of open maps, is open. Therefore, $\rho_{i}$ is a local homeomorphism.

We now show that $\Omega^{\infty}$ is Hausdorff and connected. Let $x$ and $y$ be distinct points in $\Omega^{\infty}$. Then for some $i$ there are points $x_{i} \in \rho_{i}^{-1}(x)$ and $y_{i} \in \rho_{i}^{-1}(y)$, and there are disjoint open neighborhoods $N$ of $x_{i}$ and $M$ of $y_{i}$ such that the restrictions of $\rho_{i}$ to $N$ and $M$ are homeomorphisms. Let $A=\left\{(z, w) \in \Omega_{i} \times \Omega_{i}\right.$ : $\left.\rho_{i}(z)=\rho_{i}(w)\right\}$. Then $A$ is closed in $\Omega_{i} \times \Omega_{i}$ and $(N \times M) \backslash A$ is an open neighborhood of $(x, y)$ in $\Omega_{i} \times \Omega_{i}$. Therefore, there exist open sets $U$ and $V$ in $\Omega_{i}$ such that $(x, y) \in U \times V \subset(N \times M) \backslash A$. Then $\rho_{i}(U)$ and $\rho_{i}(V)$ are disjoint open neighborhoods of $x$ and $y$ respectively. Thus $\Omega^{\infty}$ is Hausdorff. Now take any connected set $C$ in $\Omega_{i}$ containing $x_{i}$ and $y_{i}$, then $\rho_{i}(C)$ is connected and contains $x$ and $y$. It follows then that $\Omega^{\infty}$ is connected since any two points in $\Omega^{\infty}$ are contained in a connected subset of $\Omega^{\infty}$.

Let $z \in \Omega^{\infty}$. Then for some $i$ and any point $z_{i} \in \rho_{i}^{-1}(z)$, we can find a neighborhood $W_{z}$ of $z$ and a homeomorphism $\sigma_{z}: W_{z} \rightarrow \sigma_{z}\left(W_{z}\right) \subset \Omega_{i}$ (where $\sigma_{z}$ is the inverse of the restriction of $\rho_{i}$ to an appropriate neighborhood of $z$ ). From the preceding diagram we can see that $\pi^{\infty} \mid W_{z}=\pi_{i} \circ \sigma_{z}$. Since $\pi_{i}$ is a local homeomorphism, it follows then that $\pi^{\infty}$ is a local homeomorphism also.

If we give $\Omega^{\infty}$ the complex structure induced by $\pi^{\infty}$, then it is clear that $\Omega^{\infty}$ is a Riemann domain with spread $\pi^{\infty}$ and that $\rho_{i}$ is a local biholomorphism. Thus the map $\sigma_{z}$ is a biholomorphism for each $z \in \Omega^{\infty}$. Since $f^{\infty} \mid W_{z}=f_{i} \circ \sigma_{z}$ and $f_{i} \in O\left(\Omega_{i}\right)$, we have that $f^{\infty} \in \mathcal{O}\left(\Omega^{\infty}\right)$. Therefore, by the commutativity of the diagram we see that for each $i,\left(\Omega^{\infty}, f^{\infty}\right)$ is a holomorphic extension of $\left(\Omega_{i}, f_{i}\right)$. Now take distinct points $z$ and $w$ in $\Omega^{\infty}$. Then for some $i$, there are distinct points $z_{i}$ and $w_{i}$ in $\Omega_{i}$ such that $\partial^{\alpha}\left(f^{\infty}\left(\rho_{i}\left(z_{i}\right)\right)\right)=\partial^{\alpha}\left(f_{i}\left(z_{i}\right)\right)$ and $\partial^{\alpha}\left(f^{\infty}\left(\rho_{i}\left(w_{i}\right)\right)\right)=\partial^{\alpha}\left(f_{i}\left(w_{i}\right)\right) \forall \alpha$. Since $\left\{\partial^{\alpha} f_{i}: \alpha\right.$ is any multiorder $\} \cup\left\{\pi_{i j}: j=1\right.$, $\ldots, n\}$ separates points in $\Omega_{i}$, then there exists $\alpha$ such that $\partial^{\alpha}\left(f^{\infty}(z)\right) \neq$ $\partial^{\alpha}\left(f^{\infty}(w)\right)$ or there exists $j, 1 \leqslant j \leqslant n$, such that $\pi_{j}^{\infty}(z)=\pi_{i_{j}}\left(z_{i}\right) \neq \pi_{i_{j}}\left(w_{i}\right)=$ $\pi_{j}^{\infty}(w)$. Hence $\left\{\partial^{\alpha} f^{\infty}: \alpha\right.$ is any multiorder $\} \cup\left\{\pi_{j}^{\infty}: j=1, \ldots, n\right\}$ separates points in $\Omega^{\infty}$. This completes the proof of the lemma.

THEOREM 1.5. Let $\Omega$ be a Riemann domain. Then associated to each $f \in$ 
$O(\Omega)$, there is a Riemann domain $\Omega_{f}$ with spread $\pi_{f}$ and a function $\tilde{f} \in O\left(\Omega_{f}\right)$ such that $\left.\Omega_{f}\right)$;

(i) $d_{f}(z)=\tau(\tilde{f}, z)$ for every $z \in \Omega_{f}$ (where $d_{f}$ is the distance function on

(ii) $\left\{\partial^{\alpha} \tilde{f}: \alpha\right.$ is any multiorder $\} \cup\left\{\pi_{f_{j}}: j=1, \ldots, n\right\}$ separates points in $\Omega_{f}$ (where $\pi_{f_{j}}$ is the jth coordinate of $\pi_{f}$ );

(iii) the pair $\left(\Omega_{f}, \widetilde{f}\right)$ is a holomorphic extension of the pair $(\Omega, f)$. Furthermore, if $\Omega \subset \mathbf{C}^{n}$, then $\left(\Omega_{f}, \widetilde{f}\right)$ is a natural holomorphic extension of $(\Omega, f)$.

Proof. We shall first holomorphically continue $f$ by the classical method of forming power series expansions.

Let $z \in \Omega$ and let $\psi_{z}$ be the inverse of the restriction of $\pi$ to $N(z, \epsilon)$ where $\epsilon<d(z)$. The derivative $\partial^{\alpha} f(z)$ is defined by $\partial^{\alpha} f(z)=\partial^{\alpha}\left(f\left(\psi_{z}(\eta)\right)\right)$ where $\pi(z)$ $=\eta \in \Delta(\pi(z), \epsilon)$. Thus $\partial^{\alpha} f \in O(\Omega)$ and the power series expansion for $f$ about the point $z_{1}$ is given by

$$
\sum_{\alpha}\left(\pi(z)-\pi\left(z_{1}\right)\right)^{\alpha} \partial^{\alpha} f\left(z_{1}\right) / \alpha !=\sum_{\alpha}\left(\eta-\eta_{1}\right)^{\alpha} \partial^{\alpha}\left(f \circ \psi_{z_{1}}\right)\left(\eta_{1}\right) / \alpha !
$$

(where $\left.\pi\left(z_{1}\right)=\eta_{1}\right)$.

Then the power series defines a holomorphic function $f_{z_{1}}$ in $\Delta\left(\pi\left(z_{1}\right), \tau\left(f, z_{1}\right)\right)$ where $f_{z_{1}}=f \circ \psi_{z_{1}}$ in $\Delta\left(\pi\left(z_{1}\right), d\left(z_{1}\right)\right)$ and $\left(\partial^{\alpha} f\right)_{z_{1}}=\partial^{\alpha}\left(f_{z_{1}}\right)$ in $\Delta\left(\pi\left(z_{1}\right), \tau\left(f, z_{1}\right)\right)$. Denote $\Delta\left(\pi\left(z_{1}\right), \tau\left(f, z_{1}\right)\right)$ by $\Delta_{1}$ and let $\Omega+\Delta_{1}$ be the free union of $\Omega$ and $\Delta_{1}$. We define an equivalence relation $R$ in $\Omega+\Delta_{1}$ as follows:

Define $\partial^{\alpha} F: \Omega+\Delta_{1} \rightarrow C$ by

$$
\partial^{\alpha} F(z)= \begin{cases}\partial^{\alpha} f(\omega) & \text { if } \iota_{\Omega}(\omega)=z, \\ \partial^{\alpha} f_{z_{1}}(\eta) & \text { if } \iota_{\Delta_{1}}(\eta)=z,\end{cases}
$$

and define $P: \Omega+\Delta_{1} \rightarrow C^{n}$ by

$$
P(z)= \begin{cases}\pi(\omega) & \text { if } \iota_{\Omega}(\omega)=z, \\ \eta & \text { if } \iota_{\Delta_{1}}(\eta)=z,\end{cases}
$$

where $\iota_{\Omega}: \Omega \rightarrow \Omega+\Delta_{1}$ and $\iota_{\Delta_{1}}: \Delta_{1} \rightarrow \Omega+\Delta_{1}$ are the natural inclusion maps.

We define $z R w \Leftrightarrow \partial^{\alpha} F(z)=\partial^{\alpha} F(w) \forall \alpha$, and $P(z)=P(w)$. Thus

$$
\begin{aligned}
R= & \bigcap_{\alpha}\left\{(z, \omega) \in\left(\Omega+\Delta_{1}\right) \times\left(\Omega+\Delta_{1}\right): \partial^{\alpha} F(z)=\partial^{\alpha} F(w)\right\} \\
& \cap\left\{(z, w) \in\left(\Omega+\Delta_{1}\right) \times\left(\Omega+\Delta_{1}\right): P(z)=P(w)\right\} .
\end{aligned}
$$


Since $\partial^{\alpha} F(z)-\partial^{\alpha} F(w)$ and $P(z)-P(w)$ define continuous functions on $\left(\Omega+\Delta_{1}\right)$ $\times\left(\Omega+\Delta_{1}\right)$, it follows that $R$ is a closed set. We now use this fact to show that $\Omega+\Delta_{1}$ is Hausdorff.

Let $U$ be any open set in $\Omega+\Delta_{1}$ and set

$$
R U=\left\{z \in \Omega+\Delta_{1}: z R u \text { for some } u \in U\right\} .
$$

Take any $(z, u) \in R$ where $u \in U$.

Case 1. Suppose there exist $\omega$ and $\eta$ such that $\iota_{\Omega}(\omega)=z$ and $\iota_{\Delta}(\eta)=u$. Then $\pi(\omega)=\eta$ and for all $w \in N(\omega, \epsilon)$ with $\epsilon<d(\omega)$, we have that

$$
f(w)=\sum_{\alpha}(\pi(w)-\pi(\omega))^{\alpha} \partial^{\alpha} f(\omega) / \alpha !=\sum_{\alpha}(v-\eta)^{\alpha} \partial^{\alpha} f_{z_{1}}(\eta) / \alpha !
$$

where $\pi(w)=v$. If $\epsilon$ is so small that $\iota_{\Delta}(\pi(N(\omega, \epsilon))) \subset U$, then $f(w)=f_{z_{1}}(v)$ for all $w \in N(\omega, \epsilon)$ where $\iota_{\Delta}(v) \in U$ and $\pi(w)=v$. This implies that $\left(\iota_{\Omega}(w)\right) R\left(\iota_{\Delta}(v)\right)$ for all $w \in N(\omega, \epsilon)$ where $v=\pi(w)$ and $\iota_{\Delta}(v) \in U$. Set $N_{1}=$ $\iota_{\Omega}(N(\omega, \epsilon))$, then it follows that $N_{1}$ is a neighborhood of $z$ such that each point in $N_{1}$ is equivalent to some point in $U$. Thus we have that $N_{1} \subset R U$.

Case 2. Suppose there exist $\omega$ and $\eta$ such that $\iota_{\Omega}(\omega)=u$ and $\iota_{\Delta}(\eta)=z$. Then just as in Case 1 we can form the power series for $f_{\eta}$ and $f$ about $\eta$ and $\omega$ respectively. From this we see that there exists $\Delta(\eta, \epsilon) \subset \Delta_{1}$ such that for all $y \in \Delta(\eta, \epsilon), \psi_{\omega}(y) \in U$. Set $N_{2}=\iota_{\Delta}(\Delta(\eta, \epsilon))$, then $N_{2}$ is a neighborhood of $z$ such that each point in $N_{2}$ is equivalent to some point in $U$. This means that $N_{2} \subset R U$.

Case 3. Suppose there exist points $\omega_{1}$ and $\omega_{2}$ in $\Omega$ such that $\iota_{\Omega}\left(\omega_{1}\right)=z$ and $\iota_{\Omega}\left(\omega_{2}\right)=u$. Since $z R u$ we have that $P(z)=\pi\left(\omega_{1}\right)=\pi\left(\omega_{2}\right)=P(u)$ and $\partial^{\alpha} f\left(\omega_{1}\right)=\partial^{\alpha} f\left(\omega_{2}\right)=\partial^{\alpha} f\left(\psi_{\omega_{1}}(\eta)\right)=\partial^{\alpha} f\left(\psi_{\omega_{2}}(\eta)\right) \forall \alpha\left(\right.$ where $\eta=\pi\left(\omega_{1}\right)=$ $\left.\pi\left(\omega_{2}\right)\right)$. As before a consideration of the power series expansion for each shows that there exists a neighborhood of $z, N_{3} \subset \Omega+\Delta_{1}$, such that every point in $N_{3}$ is equivalent to some point in $U$.

If $\eta_{1}$ and $\eta_{2}$ are distinct points in $\Delta_{1}$, then $\iota_{\Delta}\left(\eta_{1}\right)$ and $\iota_{\Delta}\left(\eta_{2}\right)$ cannot be equivalent. Thus if $z R u$, where $u \in U$, then $z$ and $u$ must be as in Cases 1,2 or 3. Since in each case there is a neighborhood of $z$ contained in $R U$, we have that $R U$ is open in $\Omega+\Delta_{1}$. From this it follows by 4.2 of $[8$, p. 125] that the projection $p: \Omega+\Delta_{1} \rightarrow\left(\Omega+\Delta_{1}\right) / R$ is an open map. This and the fact that $R$ is closed in $\left(\Omega+\Delta_{1}\right) \times\left(\Omega+\Delta_{1}\right)$ establishes by 1.6 of $[8$, p. 140] that the quotient space $\left(\Omega+\Delta_{1}\right) / R$ is Hausdorff.

Since $P$ is constant on each fiber $p^{-1}(z)$, we can define a function $q$ : $\left(\Omega+\Delta_{1}\right) / R \rightarrow \mathrm{C}^{n}$ by $q(z)=P\left(p^{-1}(z)\right)$. By 3.2 of $[8$, p. 123] $q$ is continuous and the diagram 


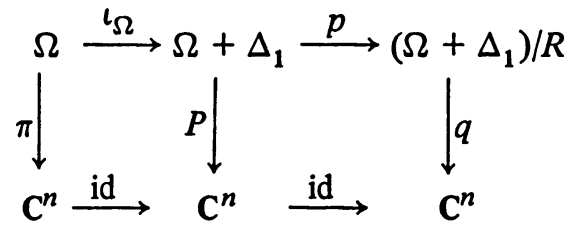

is commutative. We see that $p$ is locally injective since $P$ is a local homeomorphism. Hence $p$, being open and continuous, is a local homeomorphism also. It is clear from the diagram above, then, that $q$ is a local homeomorphism. Since $z_{1} R \eta_{1}$ (where $\left.\pi\left(z_{1}\right)=\eta_{1}\right)$ every two points in $\left(\Omega+\Delta_{1}\right) / R$ can be connected. Thus $\left(\Omega+\Delta_{1}\right) / R$ is connected. We now give $\Omega+\Delta_{1}$ and $\left(\Omega+\Delta_{1}\right) / R$ the complex structure induced by $P$ and $q$ respectively. Then $\left(\Omega+\Delta_{1}\right) / R$ is a Riemann domain with spread $q$ and all the maps in the above diagram are local biholomorphisms.

Define $\chi: \Omega \rightarrow\left(\Omega+\Delta_{1}\right) / R$ by $\chi=p \circ \iota_{\Omega}$ and define $f_{1}$ by

$$
f_{1}(z)= \begin{cases}f\left(\chi^{-1}(z)\right) & \text { if } x \in \chi(\Omega), \\ f_{z_{1}}(q(z)) & \text { if } q(z) \in \Delta_{1} .\end{cases}
$$

Then $\chi$ is a local biholomorphism and $f_{1} \in \mathcal{O}\left(\left(\Omega+\Delta_{1}\right) / R\right)$. Since $f$ is constant on each fiber $\chi^{-1}(z)$, we have that $f_{1} \circ \chi=f$ in $\Omega$ and from the preceding diagram we see that $q \circ \chi=\pi$ in $\Omega$. Hence the pair $\left(\left(\Omega+\Delta_{1}\right) / R, f_{1}\right)$ is a holomorphic extension of the pair $(\Omega, f)$. By the definition of $R$, we have that $\left\{\partial^{\alpha} f_{1}: \alpha\right.$ is any multiorder $\} \cup\left\{q_{j}: j=1, \ldots, n\right\}$ separate points in $\left(\Omega+\Delta_{1}\right) / R$ (where $q_{j}$ is the $j$ th coordinate of $q$ ). Note that there is a neighborhood of $\chi\left(z_{1}\right)$ such that the restriction of $q$ to this neighborhood is a biholomorphism onto $\Delta_{1}$ (recall $\Delta_{1}=\Delta\left(\pi\left(z_{1}\right), \tau\left(f, z_{1}\right)\right)$ ). Let $d_{1}$ be the distance function on $\left(\Omega+\Delta_{1}\right) / R$, then $d_{1}\left(\chi\left(z_{1}\right)\right)=\tau\left(f_{1}, \chi\left(z_{1}\right)\right)$.

Denote $\left(\Omega+\Delta_{1}\right) / R$ by $\Omega_{z_{1}}$ and $q$ by $\pi_{1}$. Now take a countable dense subset $\left\{z_{1}, z_{2}, \ldots\right\}$ of $\Omega$ and construct the sequence of pairs $\left\{\left(\Omega_{z_{i}}, f_{i}\right): i=\right.$ $1,2, \ldots\}$ as follows: Let $\Delta_{2}$ be the polydisc $\Delta\left(\pi\left(z_{2}\right), \tau\left(f, z_{2}\right)\right)$. Just as before we construct a holomorphic extension $\left(\Omega_{z_{2}}, f_{2}\right)$ of the pair $\left(\Omega_{z_{1}}, f_{1}\right)$ by identifying points in $\Omega_{z_{1}}+\Delta_{2}$. Thus we obtain a local biholomorphism $\chi_{1}: \Omega_{z_{1}} \rightarrow$ $\Omega_{z_{2}}$, a spread $\pi_{2}: \Omega_{z_{2}} \rightarrow \mathrm{C}^{n}$ and $f_{2} \in O\left(\Omega_{z_{2}}\right)$ such that $f_{2}^{\circ} \chi_{1}=f_{1}$ in $\Omega_{z_{1}}$ and $\pi_{2} \circ \chi_{1}=\pi_{1}$ in $\Omega_{z_{1}}$. If we let $d_{2}$ denote the distance function in $\Omega_{z_{2}}$, then $d\left(\chi_{1}\left(\chi\left(z_{1}\right)\right)\right)=\tau\left(f, z_{1}\right)$ and $d\left(\chi_{1}\left(\chi\left(z_{2}\right)\right)\right)=\tau\left(f, z_{2}\right)$, that is, $\pi_{2}$ restricted to suitable neighborhoods of $\chi_{1}\left(\chi\left(z_{1}\right)\right)$ and $\chi_{1}\left(\chi\left(z_{2}\right)\right)$, respectively, defines biholomorphisms onto $\Delta_{1}$ and $\Delta_{2}$ respectively. Then we take $z_{3}$ and similarly extend $\left(\Omega_{z_{2}}, f_{2}\right)$ to the pair $\left(\Omega_{z_{3}}, f_{3}\right), \ldots$. Thus we obtain the sequence of pairs 
$\left\{\left(\Omega_{z_{i}}, f_{i}\right): i=1,2, \ldots\right\}$ such that $\left(\Omega_{z_{i+1}}, f_{i+1}\right)$ is a holomorphic extension of $\left(\Omega_{z_{i}}, f_{i}\right)$ for each $i$. Set $\Delta_{k}=\Delta\left(\pi\left(z_{k}\right), \tau\left(f, z_{k}\right)\right)$, then the spread $\pi_{i}: \Omega_{z_{i}} \rightarrow \mathbf{C}^{n}$ restricted to suitable neighborhoods of the images of each of the points $z_{k}(1 \leqslant$ $k \leqslant i$ ), respectively, will define biholomorphisms onto each $\Delta_{k}$. Also, it is clear from the construction of $\Omega_{z_{1}}$ then, that $\left\{\partial^{\alpha} f_{i}: \alpha\right.$ is any multiorder $\} \cup\left\{\pi_{i_{j}}: j=\right.$ $1, \ldots, n\}$ separates points in $\Omega_{i}$ for all $i$. By Lemma 1.4 there exists a pair $\left(\Omega^{\infty}, f^{\infty}\right)$ which is, in particular, a holomorphic extension of $\left(\Omega_{1}, f_{1}\right)$. Since $\left(\Omega_{1}, f_{1}\right)$ extends $(\Omega, f)$, we obtain that $\left(\Omega^{\infty}, f^{\infty}\right)$ is a holomorphic extension of $(\Omega, f)$. Let $\chi^{\infty}: \Omega \rightarrow \Omega^{\infty}$ be the local biholomorphism that extends $f$ and each coordinate $\pi_{j}$ to $f^{\infty}$ and $\pi_{j}^{\infty}$ respectively. Since the distance function, $d^{\infty}$, on $\Omega^{\infty}$ is continuous and $\left\{z_{1}, z_{2}, \ldots\right\}$ is dense in $\Omega$, it follows that for any $w \in$ $\chi_{\infty}(\Omega) \subset \Omega^{\infty}, d^{\infty}(w)=\tau\left(f^{\infty}, w\right)=\tau(f, z)$ (where $\left.\chi_{\infty}(z)=w\right)$. From the second diagram in Lemma 1.4 , we see that $\left\{\partial^{\alpha} f: \alpha\right.$ is any multiorder $\} \cup\left\{\pi_{j}^{\infty}, j=1, \ldots\right.$, $n$ \} separates points in $\Omega^{\infty}$ (note that we do not need for $O(\Omega)$ or $\left\{\partial^{\alpha} f: \alpha\right.$ is any multiorder $\} \cup\left\{\pi_{j}: j=1, \ldots, n\right\}$ to separate points in $\Omega$ ).

Denote the pair $\left(\Omega^{\infty}, f^{\infty}\right)$ by $\left(\Omega_{1}^{\infty}, f_{1}^{\infty}\right)$. Now reapplying the same procedure to $\left(\Omega_{1}^{\infty}, f_{1}^{\infty}\right)$ that we applied to $(\Omega, f)$ in constructing $\left(\Omega_{1}^{\infty}, f_{1}^{\infty}\right)$, we form a sequence of pairs from which we obtain, by Lemma 1.4, a pair $\left(\Omega_{2}^{\infty}, f_{2}^{\infty}\right)$ such that $\left(\Omega_{2}^{\infty}, f_{2}^{\infty}\right)$ is a holomorphic extension of $\left(\Omega_{1}^{\infty}, f_{1}^{\infty}\right)$ and $\left\{\partial^{\alpha} f_{2}: \alpha\right.$ is any multiorder $\} \cup\left\{\pi_{2 j}^{\infty}: j=1, \ldots, n\right\}$ (where $\pi_{2 j}$ is the $j$ th coordinate of the spread $\pi_{2}^{\infty}$ : $\Omega_{2}^{\infty} \rightarrow \mathrm{C}^{n}$ ) separates points in $\Omega_{2}^{\infty}$. Iterating we obtain the sequence of pairs $\left\{\left(\Omega_{i}^{\infty}, f_{i}^{\infty}\right): i=1,2, \ldots\right\}$ such that, for each $i,\left(\Omega_{i+1}^{\infty}, f_{i+1}^{\infty}\right)$ extends $\left(\Omega_{i}^{\infty}, f_{i}^{\infty}\right)$ and $\left\{\partial^{\alpha} f_{i}^{\infty}: \alpha\right.$ is any multiorder $\} \cup\left\{\pi_{i j}^{\infty}: j=1, \ldots, n\right\}$ separates points in $\Omega_{i}$ $\left(\pi_{i j}^{\infty}\right.$ is the $j$-coordinate of the spread $\left.\pi_{i}^{\infty}: \Omega_{i}^{\infty} \rightarrow \mathbf{C}^{n}\right)$. Hence it follows from Lemma 1.4 that there exists a pair $\left(\Omega_{f}, \widetilde{f}\right)$ such that $\left\{\partial^{\alpha} \widetilde{f}: \alpha\right.$ is any multiorder $\}$ $\cup\left\{\pi_{f_{j}}: j=1, \ldots, n\right\}$ separates points in $\Omega_{f}$ (where $\pi_{f_{j}}$ is the $j$-coordinate of the spread $\left.\pi_{f}: \Omega_{f} \rightarrow \mathbf{C}^{n}\right)$, and $\left(\Omega_{f}, \widetilde{f}\right)$ is a holomorphic extension of $\left(\Omega_{i}^{\infty}, f_{i}\right)$ for each $i$. Since $\left(\Omega_{1}^{\infty}, f_{1}\right)$ is a holomorphic extension of $(\Omega, f)$, we have that $\left(\Omega_{f}, \widetilde{f}\right)$ is a holomorphic extension of $(\Omega, f)$. Thus there exists a local biholomorphism $\chi_{f}: \Omega \rightarrow \Omega_{f}$ such that $\widetilde{f} \circ \chi_{f}=f$ in $\Omega$ and $\pi_{f} \circ \chi_{f}=\pi$ in $\Omega$.

If $d_{f}(z)<\tau(\widetilde{f}, z)$ for some $z \in \Omega_{f}$, then for some $i$ there exists a point $w \in \Omega_{i}^{\infty}$, where $\rho_{i}(w)=z\left(\rho_{i}\right.$ is defined in Lemma 1.4), such that the power series for $\widetilde{f}$ at $z, \Sigma_{\alpha}\left(\eta-\tau_{f}(z)\right)^{\alpha} \partial^{\alpha} \widetilde{f}(z) / \alpha$ !, converges in $\Delta\left(\pi_{f}(z), \tau(\widetilde{f}, z)\right)$ and coincides with the power series for $f_{i}^{\infty}$ at $w, \Sigma_{\alpha}\left(\eta-\pi_{i}^{\infty}(w)\right)^{\alpha} \partial^{\alpha} f_{i}^{\infty}(w) / \alpha !$, since $\pi_{f}(z)=$ $\pi_{i}^{\infty}(w)$. Now there exist neighborhoods $N$ of $z$ and $M$ of $w$ such that the inverses of the restrictions of $\pi_{f}$ and $\pi_{i}^{\infty}$ to $N$ and $M$, respectively, and $\rho_{i}$ restricted to $M$ are biholomorphisms and hence

$$
\partial^{\alpha} \widetilde{f}(z)=\partial^{\alpha} \tilde{f}\left(\pi_{f}^{-1}(\lambda)\right)=\partial^{\alpha} \tilde{f}\left(\rho_{i}\left(\pi_{i}^{\infty-1}(\lambda)\right)\right)=\partial^{\alpha} f_{i}\left(\pi_{i}^{\infty-1}(\lambda)\right)=\partial^{\alpha} f_{i}(w)
$$


(where $\left.\lambda=\pi_{f}(z)=\pi_{i}^{\infty}(w)\right)$. This means that $\Delta\left(\pi_{f}(z), \tau(\tilde{f}, z)\right) \subset \pi_{i+1}^{\infty}\left(\Omega_{i+1}^{\infty}\right)$. By the construction of $\Omega_{f}$, it follows that $\Delta\left(\pi_{f}(z), \tau(\widetilde{f}, z)\right) \subset \pi_{f}\left(\Omega_{f}\right)$, which is a contradiction. Since $\tilde{f} \in O\left(\Omega_{f}\right)$, we can conclude that $d_{f}(z)=\tau(\widetilde{f}, z) \forall z \in \Omega_{f}$.

If $\Omega$ is a domain in $\mathbf{C}^{n}$ with spread the identity map on $\mathbf{C}^{n}$, then the map $\chi_{f}: \Omega \rightarrow \Omega_{f}$ is injective since $\pi_{f}$ restricted to $\chi_{f}(\Omega)$ is injective. This completes the proof.

REMARKS. Even if $O(\Omega)$ separates points in $\Omega,\left(\Omega_{f}, \widetilde{f}\right)$ may not be a natural holomorphic extension of the pair $(\Omega, f)$, for example, $\Omega$ may be nonschlicht but $\Omega_{f} \subset \mathbf{C}^{n}$. So nothing is gained in Theorem 1.5 when $O(\Omega)$ separates points in $\Omega$. A Riemann domain is said to be nonschlicht whenever its spread is not injective.

By Theorem 7 in $[9$, p. 47] we have

LEMMA 1.6. Let $\Omega$ be a Riemann domain and let $f \in O(\Omega)$. Then for any compact set $K \subset \Omega_{f}, d_{f}\left(\hat{K}_{\Omega_{f}}\right)=d_{f}(K)$.

Theorem 17 in $\left[9\right.$, p. 54] shows that $\Omega_{f}$ is holomorphically convex. Thus $\Omega_{f}$ is a Stein manifold.

REMARK. The proof of Theorem 17 of $[9$, p. 54] does not depend on a solution of the Levi problem and is based on the work of Bishop in [2].

II. Envelope of holomorphy of a Riemann domain. We define the notion of envelope of holomorphy of a Riemann domain $\Omega$ by properties it is to have. Later we offer an alternate definition.

Definition 2.1. Let $\Omega$ be a Riemann domain with spread $\pi: \Omega \rightarrow \mathbf{C}^{n}$. Then any Riemann domain $\widetilde{\Omega}$ with spread $\tilde{\pi}: \Omega \rightarrow \mathbf{C}^{n}$ is called an envelope of holomorphy of $\Omega$ if $\widetilde{\Omega}$ is a Stein manifold and if there exists a local biholomorphism $\chi: \Omega \rightarrow \widetilde{\Omega}$ such that

(i) $\tilde{\pi} \circ \chi=\rho$ in $\Omega$, and

(ii) for each $f \in O(\Omega)$ there is a function $\tilde{f} \in O(\widetilde{\Omega})$ such that $\widetilde{f} \circ \chi=f$ in $\Omega$.

REMARKS. It is common to speak of the envelope of holomorphy since envelopes of holomorphy of the same Riemann domain are biholomorphic, however, here we use the article an to emphasize different representations. If in 2.1 we were to require that $O(\Omega)$ separate points in $\Omega$, which is usually done, then the map $\chi: \Omega \rightarrow \chi(\Omega) \subset \widetilde{\Omega}$ would be a biholomorphism onto $\chi(\Omega)$.

We now construct an envelope of holomorphy for a Riemann domain $\Omega$ from the family $\left\{\Omega_{f}: f \in O(\Omega)\right\}$ of "Riemann surfaces" of functions holomorphic in $\Omega$.

By Theorem 1.5 for any Riemann domain $\Omega$ with spread $\pi$ and any $f \in O(\Omega)$ there exist a Riemann domain $\Omega_{f}$ with spread $\pi_{f}$ and a local biholomorphism $\chi_{f}$ : 
$\Omega \rightarrow \Omega_{f}$ such that $\pi_{f}^{\circ} \chi=\pi$ in $\Omega$. We use this to define a preordering in $O(\Omega)$.

Definition 2.2. For $f$ and $g \in O(\Omega)$, we define $g<f$ if and only if there exists a local biholomorphism $\psi_{f g}: \Omega_{f} \rightarrow \Omega_{g}$ such that the diagram

is commutative.

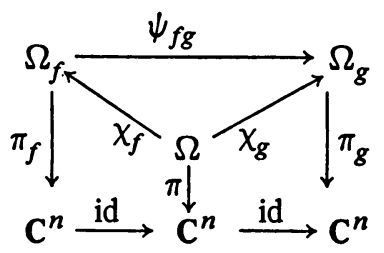

Lemma 2.3. If $h<g<f$, then $\psi_{f h}=\psi_{h g} \circ \psi_{f g}$ in $\Omega_{f}$.

Proof. From the diagram we see that $\left(\psi_{h g} \circ \psi_{f g}\right) \circ \chi_{f}=\psi_{h g} \circ \chi_{g}=\chi_{h}$ in $\Omega$ and $\psi_{g h}{ }^{\circ} \chi_{f}=\chi_{h}$ in $\Omega$. Thus $\psi_{f h}=\psi_{h g}{ }^{\circ} \psi_{f g}$ in $\chi_{f}(\Omega)$, an open connected set in $\Omega_{f}$. Hence, by the uniqueness of analytic continuation, $\psi_{f h}=\psi_{h g}{ }^{\circ} \psi_{f g}$ in $\Omega_{f}$.

Lemma 2.3 implies that the family $\left\{\Omega_{f} ; \psi_{f g}\right\}$ is a projective spectrum over $O(\Omega)$ with spaces $\Omega_{f}$ and connecting maps $\psi_{f g}$, where $O(\Omega)$ is preordered by $<$. The notion of projective (inverse) spectrum is defined in [8, p. 427].

Definition 2.4. The topological space $\left(\Pi_{f} \Omega_{f}, S\right)$ is the cartesian product over $O(\Omega)$ of the sets $\Omega_{f}$ with topology determined by the basis $\left\{\Pi_{f} u_{f}:\right.$ for each $f \in O(\Omega), u_{f}$ is an open set in the Riemann domain $\Omega_{f}$ and for each $y \in \Pi_{f} u_{f}$, there exist $\theta_{y}>0$ and neighborhoods $N\left(p_{g}(y), \theta_{y}\right)$ of $p_{g}(y)$ for each $g \in O(\Omega)$ such that $N\left(p_{g}(y), \theta_{y}\right) \subset u_{g}$ and the restriction of each $\pi_{g}$ to $N\left(p_{g}(y), \theta_{y}\right)$ is a biholomorphism onto each polydisc $\Delta\left(\pi_{g} \circ p_{g}(y), \theta_{y}\right)$ \}, where $\pi_{g}$ is the spread of $\Omega_{g}$ into $\mathbf{C}^{n}$ and $p_{g}:\left(\Pi_{f} \Omega_{f}, S\right) \rightarrow \Omega_{g}$ is projection onto the gth factor.

We now consider the projective limit of the projective spectrum $\left\{\Omega_{f}: \psi_{f g}\right\}$ with the induced topology from $\left(\Pi_{f} \Omega_{f}, S\right)$.

Definition 2.5. We define the $S$-projective limit space, $S(\Omega)$, of the projective spectrum $\left\{\Omega_{f} ; \psi_{f g}\right\}$ as the subspace of $\left(\Pi_{f} \Omega_{f}, S\right)$,

$$
S(\Omega)=\left\{y \in \prod_{f} \Omega_{f}: \text { if } g<f, \text { then } p_{g}(y)=\psi_{f g} \circ p_{f}(y)\right\} .
$$

We let $\widetilde{\Omega}$ be the projective limit space of the projective spectrum $\left\{\Omega_{f} ; \psi_{f g}\right\}$, thus $\widetilde{\Omega}$ is the set $S(\Omega)$ with the topology induced by the cartesian product topology in $\Pi_{f} \Omega_{f}$.

Definition 2.6. For each $f \in O(\Omega)$, let $p_{f}$ denote projection onto $\Omega_{f}$ and let $d_{f}$ be the distance function on $\Omega_{f}$. We define $d_{\infty}: S(\Omega) \rightarrow R^{+}$(nonnegative reals) by

$$
d_{\infty}(y)=\inf _{f \in O(\Omega)} d_{f}^{\circ} p_{f}(y) .
$$


Let $S^{0}(\Omega)=\left\{y \in S(\Omega): d_{\infty}(y)>0\right\}$. Then for any $z \in S^{0}(\Omega)$ there exists $\theta_{z}>$ 0 such that $d_{f} \circ p_{f}(z) \geqslant \theta_{z} \forall f \in O(\Omega)$.

DEFINITION 2.7. Let $E(\Omega)$ be the subspace of $S(\Omega)$ defined by

$$
E(\Omega)=\left\{y \in S^{0}(\Omega): \pi_{f}^{\circ} p_{f}(y)=\pi_{g} \circ p_{g}(y) \forall f, g \in O(\Omega)\right\} .
$$

We denote by $\widetilde{E}$ the set $E(\Omega)$ with the topology induced by $\Pi_{f} \Omega_{f}$. Then the topology in $E(\Omega)$ is stronger than the topology in $\widetilde{E}$. To see this let $u_{f}$ be open in $\Omega_{f}$ for each $f \in O(\Omega)$, where $u_{f}=\Omega_{f}$ except for finitely many $f$. Then $E(\Omega) \cap \Pi_{f} u_{f}$ is open in $E(\Omega)$ since for each $y \in E(\Omega) \cap \Pi_{f} u_{f}$ there exists $\theta_{y}$ such that $d_{f}^{\circ} p_{f}(y) \geqslant \theta_{y} \forall f \in O(\Omega)$. Thus, since the restriction of $p_{f}$ to $\widetilde{E}$ is continuous, we have that the restriction of $p_{f}$ to $E(\Omega)$, which we denote by $\psi_{f}$, is also continuous.

$\widetilde{E}$, being a subspace of $\Pi_{f} \Omega_{f}$, is Hausdorff since each $\Omega_{f}$ is Hausdorff. Therefore $E(\Omega)$ is a Hausdorff space. We now show that $E(\Omega)$ is a complex analytic manifold of dimension $n$.

From the maps $\chi_{f}: \Omega \rightarrow \Omega_{f}$, we define the function $\chi_{E}: \Omega \rightarrow \Pi_{f} \Omega_{f}$ by $\chi_{E}(z)=\left\{\chi_{f}(z)\right\} \in \Pi_{f} \Omega_{f}$. We now show that $E(\Omega) \neq \varnothing$.

LEMMA 2.8. $\chi_{E}(\Omega) \subset E(\Omega)$.

Proof. Take any $z \in \Omega$. Then for each $f \in O(\Omega)$, we have $d_{f}\left(\chi_{f}(z)\right) \geqslant$ $d(z)>0$ where $d$ is the distance function on $\Omega$. Thus $d_{\infty}\left(\chi_{E}(z)\right)=\inf _{f} d_{f}\left(\chi_{f}(z)\right)$ $\geqslant d(z)>0$. This means that $\chi_{E}(z) \in S^{0}(\Omega)$. We see from the diagram in Definition 2.2 that $\forall f, g \in O(\Omega)$,

$$
\pi_{f}^{\circ} p_{f}\left(\chi_{E}(z)\right)=\pi_{f}\left(\chi_{f}(z)\right)=\pi(z)=\pi_{g}\left(\chi_{g}(z)\right)=\pi_{g} \circ p_{g}\left(\chi_{E}(z)\right) .
$$

Therefore $\chi_{E}(z) \in E(\Omega)$, which completes the proof.

Note that Lemma 2.8 means that $\chi_{E}: \Omega \rightarrow E(\Omega) \subset \Pi_{f} \Omega_{f}$.

From the definition of $E(\Omega)$ (2.6) we have that for each fixed $z \in E(\Omega)$, $\pi_{f} \circ \psi_{f}(z)$ is constant $\forall f \in O(\Omega)$. We define the function $\pi_{E}: E(\Omega) \rightarrow \mathrm{C}^{n}$ by

$$
\pi_{E}(y)=\pi_{f} \circ \psi_{f}(y) \text { for any } f \in O(\Omega) .
$$

Then $\pi_{E}$ is continuous since $\pi_{f}$ and $\psi_{f}$ are continuous. It is clear that $\pi_{E}{ }^{\circ} \chi_{E}=$ $\pi$ in $\Omega$. Hence the diagram

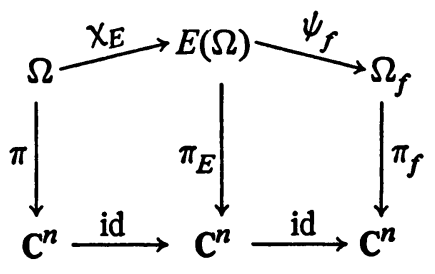

is commutative for each $f \in O(\Omega)$. 


\section{LEMMA 2.9. $\pi_{E}$ is a local homeomorphism.}

Proof. Take any $z \in E(\Omega)$ and any neighborhood of $z, U$. We find a neighborhood of $z$ such that $\pi_{E}$ restricted to this neighborhood is injective and open.

There exists $\theta_{z}>0$ such that for each $f \in O(\Omega)$, there is a neighborhood $N_{f}$ of $\psi_{f}(z)$ such that the restriction of $\pi_{f}$ to $N_{f}$ is a homeomorphism onto $\Delta\left(\pi_{f} \circ \psi_{f}(z), \theta_{z}\right)$, where $E(\Omega) \cap \Pi_{f} N_{f} \subset U$. Let $V=E(\Omega) \cap \Pi_{f} N_{f}$; then $V \subset$ $U$ is an open neighborhood of $z$ in $E(\Omega)$ and $\pi_{E}$ is injective on $V$. This is true because if $\pi_{E}\left(y_{1}\right)=\pi_{E}\left(y_{2}\right)$ and $y_{1}, y_{2} \in V$, then $\pi_{f} \circ \psi_{f}\left(y_{1}\right)=\pi_{f} \circ \psi_{f}\left(y_{2}\right)$ for each $f$. Since $\psi_{f}\left(y_{1}\right), \psi_{f}\left(y_{2}\right) \in N_{f}$ and $\pi_{f}$ is injective on $N_{f}$, we would have that $\psi_{f}\left(y_{1}\right)=\psi_{f}\left(y_{2}\right)$ for any $f \in O(\Omega)$. This means that $y_{1}=y_{2}$.

Now we show that $\pi_{E}(V)$ is open in $\mathbf{C}^{n}$. For any $\eta \in \Delta\left(\pi_{E}(z), \theta_{z}\right)$ there is a unique point $w_{f} \in N_{f}$, for each $f$, such that $\pi_{f}\left(w_{f}\right)=\eta$. Let $w=\left\{w_{f}\right\} \in$ $\Pi_{f} N_{f}$. Then $w \in E(\Omega)$. To see this consider any $f, g \in O(\Omega)$ such that $g<f$. Then $\psi_{f g}\left(\psi_{f}(z)\right)=\psi_{g}(z)$ and $\psi_{f g}\left(N_{f}\right)=N_{g}$, in fact, $\psi_{f g}$ restricted to $N_{f}$ is a biholomorphism onto $N_{g}$. It follows then that $\psi_{f g}\left(w_{f}\right)=\psi_{f g}\left(w_{g}\right)$ since $\pi_{f}$ and $\pi_{g}$ are injective on $N_{f}$ and $N_{g}$, respectively, and $\pi_{f}\left(w_{f}\right)=\pi_{g}\left(w_{g}\right)$ with $w_{f} \in N_{f}$ and $w_{g} \in N_{g}$. Thus for each $\eta$ in the polydisc $\Delta\left(\pi_{E}(z), \theta_{z}\right)$ there exists $w \in V$ such that $\pi_{E}(w)=\eta$. This means that $\pi_{E}(V)=\Delta\left(\pi_{E}(z), \theta_{z}\right)$, an open set in $\mathbf{C}^{n}$. This shows that $\pi_{E}$ is an open map and is locally injective. Since $\pi_{E}$ is continuous, we can conclude that $\pi_{E}$ is a local homeomorphism, which completes the proof.

From the preceding diagram, we see that $\chi_{E}$ is a local homeomorphism also. We give $E(\Omega)$ the complex structure induced by $\pi_{E}$. Then $E(\Omega)$ is an $n$-dimensional complex analytic manifold and $\pi_{E}$ is a local biholomorphism. Hence $\chi_{E}$ is a local biholomorphism.

Since $\Omega$ is a Riemann domain, we have that $\chi_{E}(\Omega)$ is an open connected set in $E(\Omega)$. Let $E^{\prime}(\Omega)$ be the component of $E(\Omega)$ containing $\chi_{E}(\Omega)$. Then $E^{\prime}(\Omega)$ is open in $E(\Omega)$ because $E(\Omega)$ is locally connected. Let $\pi_{E^{\prime}}, \chi_{E^{\prime}}$ and $\varphi_{f}^{\prime}$ be the restrictions to $E^{\prime}(\Omega)$ of $\pi_{E}, \chi_{E}$ and $\psi_{f}$, respectively. Then $E^{\prime}(\Omega)$ is a Riemann domain with spread $\pi_{E^{\prime}}$ and the diagram

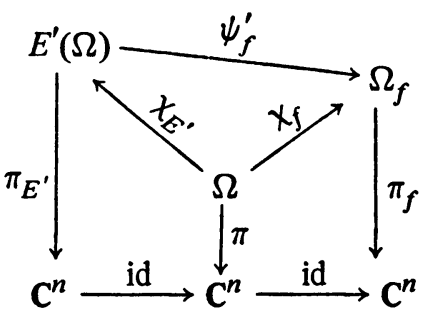

is commutative for each $f \in O(\Omega)$. Furthermore all maps in the diagram are local biholomorphisms. 
LEMma 2.10. For each $f \in O(\Omega)$ there exists $f_{E^{\prime}} \in O\left(E^{\prime}(\Omega)\right)$ such that $f_{E^{\prime}} \circ \chi_{E^{\prime}}=f$ in $\Omega$.

Proof. Take any $f \in O(\Omega)$. By Theorem 1.5 there exists $\tilde{f} \in O\left(\Omega_{f}\right)$ such that $\widetilde{f} \circ \chi_{f}=f$ in $\Omega$. Let $f_{E^{\prime}}=\widetilde{f} \circ \psi_{f}^{\prime}$. Then $f_{E^{\prime}} \in O\left(E^{\prime}(\Omega)\right)$ and

$$
f_{E^{\prime}} \circ \chi_{E^{\prime}}=\tilde{f} \circ\left(\psi_{f}^{\prime} \circ \chi_{E^{\prime}}\right)=\tilde{f} \circ \chi_{f}=f
$$

in $\Omega$, which proves the lemma.

Lemma 2.11. $O\left(E^{\prime}(\Omega)\right.$ ) separates points in $E^{\prime}(\Omega)$.

Proof. Take any two distinct points $z_{1}$ and $z_{2}$ in $E^{\prime}(\Omega)$. Then for some $f \in O(\Omega), \psi_{f}^{\prime}\left(z_{1}\right) \neq \psi_{f}^{\prime}\left(z_{2}\right)$. From Theorem 1.5 we have that $O\left(\Omega_{f}\right)$ separates points in $\Omega_{f}$. Thus there exists $h \in O\left(\Omega_{f}\right)$ such that $h\left(\psi_{f}^{\prime}\left(z_{1}\right)\right) \neq h\left(\psi_{f}^{\prime}\left(z_{2}\right)\right)$. Since $h \circ \psi_{f}^{\prime} \in O\left(E^{\prime}(\Omega)\right)$, the lemma is proved.

Let $d_{\infty}^{\prime}$ be the restriction of $d_{\infty}$ to $E^{\prime}(\Omega)$, where $d_{\infty}$ is defined as in Definition 2.6.

LEMMA 2.12. $d_{\infty}^{\prime}$ is the distance function on $E^{\prime}(\Omega)$.

Proof. Let $d_{E^{\prime}}$ be the distance function on $E^{\prime}$. We show that $d_{E^{\prime}}=d_{\infty}^{\prime}$. Take any $z \in E^{\prime}(\Omega)$. Let $d_{\infty}^{\prime}(z)=\theta_{z}>0$ and let $d_{E^{\prime}}(z)=\varphi_{z}>0$. By the definition of $d_{\infty}^{\prime}$, we have that for each $f \in O(\Omega), d_{f} \circ \psi_{f}^{\prime}(z) \geqslant \theta_{z}$, where $d_{f}$ is the distance function on $\Omega_{f}$. This means that for each $f$, there exists a neighborhood $N_{f}$ of $\psi_{f}^{\prime}(z)$ such that $\pi_{f}$ restricted to $N_{f}$ is a biholomorphism onto $\Delta\left(\pi_{E^{\prime}}(z), \theta_{z}\right)$. Thus $V=E^{\prime}(\Omega) \cap \Pi_{f} N_{f}$ is a neighborhood of $z$ such that $\pi_{E^{\prime}}$ restricted to $V$ is a biholomorphism onto $\Delta\left(\pi_{E^{\prime}}(z), \theta_{z}\right)$. This implies that $\varphi_{z} \geqslant \theta_{z}$.

However, since $d_{E^{\prime}}(z)=\varphi_{z}$ there is a neighborhood $U$ of $z$ such that $\pi_{E^{\prime}}$, restricted to $U$, is a biholomorphism onto $\Delta\left(\pi_{E^{\prime}}(z), \varphi_{z}\right)$. Then for each $f, \psi_{f}^{\prime}(U)$ is open in $\Omega_{f}$ and $\pi_{f}$ restricted to $\psi_{f}^{\prime}(U)$ is a biholomorphism onto $\Delta\left(\pi_{E^{\prime}}(z), \varphi_{z}\right)$. This means that $d_{f} \circ \psi_{f}^{\prime}(z) \geqslant \varphi_{z} \forall f \in O(\Omega)$. Therefore $\theta_{z} \geqslant \varphi_{z}$. We conclude that $\theta_{z}=\varphi_{z}$, which proves the lemma.

$d_{\infty}^{\prime}$, being the distance function on $E^{\prime}(\Omega)$, is continuous. We now show that $E^{\prime}(\Omega)$ is holomorphically convex.

Let $K$ be a compact subset of $E^{\prime}(\Omega)$. Let $\hat{K}_{E^{\prime}(\Omega)}$ denote the $O\left(E^{\prime}(\Omega)\right)$-hull of $K$ and for each $f \in O(\Omega)$, let $\widehat{\psi_{f}^{\prime}(K)_{\Omega_{f}}}$ denote the $O\left(\Omega_{f}\right)$-hull of $\psi_{f}^{\prime}(K)$.

LEMMA 2.13. For any compact set $\left.K \subset E^{\prime}(\Omega), \psi_{f}^{\prime}\left(\hat{K}_{E^{\prime}(\Omega)}\right) \subset \widehat{\psi_{f}^{\prime}(K}\right)_{\Omega_{f}}$ for each $f \in O(\Omega)$.

Proof. For any $f \in O(\Omega)$, take any point $\psi_{f}^{\prime}(z) \in \psi_{f}^{\prime}\left(\hat{K}_{E^{\prime}(\Omega)}\right)$. Then for each $g_{E^{\prime}} \in O\left(E^{\prime}(\Omega)\right)$ we have that $\left|g_{E^{\prime}}(z)\right| \leqslant \sup _{K}|g|$. Now for each $\widetilde{h} \in O\left(\Omega_{f}\right)$, 
$\widetilde{h} \circ \psi_{f}^{\prime} \in O\left(E^{\prime}(\Omega)\right)$. Hence for every such $\widetilde{h}$,

$$
\widetilde{h}\left(\psi_{f}^{\prime}(z)\right)\left|\leqslant \sup _{K}\right| \widetilde{h} \circ \psi_{f}^{\prime}\left|=\sup _{\psi_{f}^{\prime}(K)}\right| \widetilde{h} \mid .
$$

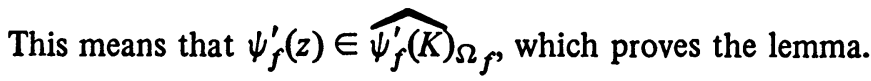

Lemma 2.14. For any compact set $K \subset E^{\prime}(\Omega), d_{\infty}^{\prime}(K)=d_{\infty}^{\prime}\left(\hat{R}_{E^{\prime}(\Omega)}\right)$.

ProOF. From the preceding lemma we have that for each $f \in O(\Omega), \psi_{f}^{\prime}(K)$ $\subset \psi_{f}^{\prime}\left(\hat{K}_{E^{\prime}(\Omega)}\right) \subset \widehat{\psi}_{f}^{\prime}(K)_{\Omega_{f}}$. This implies that for each $f, d_{f}\left(\psi_{f}^{\prime}(K)\right) \geqslant d_{f}\left(\psi_{f}^{\prime}\left(R_{E^{\prime}(\Omega)}\right)\right)$

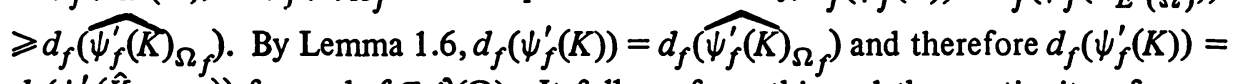
$d_{f}\left(\psi_{f}^{\prime}\left(\hat{K}_{E^{\prime}(\Omega)}\right)\right)$ for each $f \in O(\Omega)$. It follows from this and the continuity of $d_{\infty}^{\prime}$ that

$$
d_{\infty}^{\prime}(K)=\inf _{f} d_{f} \circ \psi_{f}(K)=\inf _{f} d_{f} \circ \psi_{f}\left(\hat{K}_{E^{\prime}(\Omega)}\right)=d_{\infty}^{\prime}\left(R_{E^{\prime}(\Omega)}\right) .
$$

This proves the lemma.

From the remark preceding Definition 1.2, $E^{\prime}(\Omega)$ is countable at infinity and Theorem 17 of $\left[9\right.$, p. 54] shows that $E^{\prime}(\Omega)$ is holomorphically convex. Hence $E^{\prime}(\Omega)$ is a Stein manifold. This together with Lemmas 2.10 and 2.11 and the preceding diagram establish

THEOREM 2.15. Let $\Omega$ be a Riemann domain. Then $E^{\prime}(\Omega)$ is an envelope of holomorphy for $\Omega$.

COROLlaRY 2.16. If $\Omega$ is a Riemann domain such that $O(\Omega)$ separates points in $\Omega$, then $\chi_{E^{\prime}}: \Omega \rightarrow \chi_{E^{\prime}}(\Omega) \subset E^{\prime}(\Omega)$ is a biholomorphism onto $\chi_{E^{\prime}}(\Omega)$.

Proof. Let $z_{1}$ and $z_{2}$ be any two distinct points in $\Omega$. Then there exists $f \in O(\Omega)$ such that $f\left(z_{1}\right) \neq f\left(z_{2}\right)$. Let $f_{E^{\prime}}$ be the unique function in $O\left(E^{\prime}(\Omega)\right.$ ) such that $f_{E^{\prime}}^{\circ} \chi_{E^{\prime}}=f$ in $\Omega$. Thus

$$
f\left(z_{1}\right)=f_{E^{\prime}}\left(\chi_{E^{\prime}}\left(z_{1}\right)\right) \neq f_{E^{\prime}}\left(\chi_{E^{\prime}}\left(z_{2}\right)\right)=f\left(z_{2}\right),
$$

which implies that $\chi_{E^{\prime}}\left(z_{1}\right) \neq \chi_{E^{\prime}}\left(z_{2}\right)$. This proves the lemma.

We now show that $\widetilde{\Omega}$ (as defined in 2.5 ) is an envelope of holomorphy for $\Omega$.

LEMmA 2.17. Let $\Omega$ be a Riemann domain. Then there exists a function $h \in O(\Omega)$ such that the map $\psi_{h}^{\prime}: E^{\prime}(\Omega) \rightarrow \Omega_{h}$ is a biholomorphism onto $\Omega_{h}$.

Proof. Since $E^{\prime}(\Omega)$ is a holomorphically convex Riemann domain there exists $h_{E^{\prime}} \in O\left(E^{\prime}(\Omega)\right)$ such that $d_{\infty}^{\prime}(z)=\tau\left(h_{E^{\prime}}, z\right)$ for all $z \in E^{\prime}(\Omega)$. Just as in Theorem 1.5 we form $\left(E^{\prime}(\Omega)\right)_{n_{E^{\prime}}}$. Since $E^{\prime}(\Omega)$ separates points in $E^{\prime}(\Omega)$ and 
$d_{\infty}^{\prime}(z)=\tau\left(h_{E^{\prime}}, z\right) \forall z \in E^{\prime}(\Omega)$, we have that $\left(E^{\prime}(\Omega)\right)_{h_{E^{\prime}}}=E^{\prime}(\Omega)$ and $\left\{\partial^{\alpha} h_{E^{\prime}}: \alpha\right.$ is any multiorder $\} \cup\left\{\pi_{E_{j}^{\prime}}: j=1, \ldots, n\right\}$ separates points in $E^{\prime}(\Omega)$, where $\pi_{E_{j}^{\prime}}$ is the $j$ th coordinate of $\pi_{E^{\prime}}$. Let $h=h_{E^{\prime}} \circ \chi_{E^{\prime}} \in O(\Omega)$. By Theorem 1.5 there exists $\Omega_{h}$ and $\tilde{h} \in O\left(\Omega_{h}\right)$ such that $\widetilde{h} \circ \chi_{h}=h$ in $\Omega$. Hence, by the principle of analytic continuation, $\widetilde{h} \circ \psi_{h}^{\prime}=h_{E^{\prime}}$ in $E^{\prime}(\Omega)$ and $\left(\partial^{\alpha} \widetilde{h}\right) \circ \psi_{h}^{\prime}=\partial^{\alpha} h_{E^{\prime}}$ in $E^{\prime}(\Omega)$ for any multiorder $\alpha$. Take any two distinct points $z_{1}$ and $z_{2}$ in $E^{\prime}(\Omega)$. Then either $\partial^{\alpha} h_{E^{\prime}}\left(z_{1}\right) \neq \partial^{\alpha} h_{E^{\prime}}\left(z_{2}\right)$ for some multiorder $\alpha$ or $\pi_{E_{j}^{\prime}}\left(z_{1}\right) \neq \pi_{E_{j}^{\prime}}\left(z_{2}\right)$ for some $j=1, \ldots, n$. Since $\pi_{E^{\prime}}=\pi \circ \psi_{h}^{\prime}$, it follows that either $\left(\partial^{\alpha} \widetilde{h}\right) \circ$ $\psi_{h}^{\prime}\left(z_{1}\right) \neq\left(\partial^{\alpha} \tilde{h}\right) \circ \psi_{h}^{\prime}\left(z_{2}\right)$ for some multiorder $\alpha$ or $\pi_{h} \circ \psi_{h}^{\prime}\left(z_{1}\right) \neq \psi_{h} \circ \psi_{h}^{\prime}\left(z_{2}\right)$. Now either case implies that $\psi_{h}^{\prime}\left(z_{1}\right) \neq \psi_{h}^{\prime}\left(z_{2}\right)$. This establishes that $\psi_{h}^{\prime}$ is injective.

We now show that $\psi_{h}^{\prime}$ is surjective. Suppose that $\psi_{h}^{\prime}\left(E^{\prime}(\Omega)\right) \neq \Omega_{h}$. Then $\psi_{h}^{\prime}\left(E^{\prime}(\Omega)\right)$ is open in $\Omega_{h}$ and there exists $z \in \Omega_{h} \cap \partial\left(\psi_{h}^{\prime}\left(E^{\prime}(\Omega)\right)\right)$. Let $\left\{z_{n}\right\}$ be a sequence in $\psi_{h}^{\prime}\left(E^{\prime}(\Omega)\right)$ converging to $z$. For each $n=1,2, \ldots$ let $w_{n}=$ $\left(\psi_{h}^{\prime}\right)^{-1}\left(z_{n}\right)$ (this is well defined since $\psi_{h}^{\prime}$ is injective). Since $\tilde{h} \circ \psi_{h}^{\prime}=h_{E^{\prime}}$, we have that $d_{\infty}^{\prime}\left(w_{n}\right)=\tau\left(h_{E^{\prime}}, w_{n}\right)=\tau\left(\tilde{h}, z_{n}\right)=d_{h}\left(z_{n}\right)$ for all $n$. Now $\lim _{n \rightarrow \infty} d_{h}\left(z_{n}\right)$ $=d_{h}(z)>0$ since $z \in \Omega_{h}$; on the other hand $\lim _{n \rightarrow \infty} d_{\infty}^{\prime}\left(w_{n}\right)=0$. This contradicts the fact that $d_{\infty}^{\prime}\left(w_{n}\right)=d_{h}\left(z_{n}\right)$ for all $n$. Hence $\psi_{h}^{\prime}\left(E^{\prime}(\Omega)\right)=\Omega_{h}$. Since $\psi_{h}^{\prime}$ is a local biholomorphism, we have that $\psi_{h}^{\prime}$ is a biholomorphism onto $\psi_{h}$. This completes the proof. Note that $\left\{\partial^{\alpha} h_{E^{\prime}}: \alpha\right.$ is any multiorder $\} \cup\left\{\pi_{E_{j}^{\prime}}: j=1\right.$, $\ldots, n$ \} separates points in $E^{\prime}(\Omega)$.

Lemma 2.17 together with Theorem 2.15 imply that $\Omega_{h}$ is an envelope of holomorphy for $\Omega$. Thus any Riemann domain has an envelope of holomorphy which is a direct limit of Riemann domains. For a construction of an envelope of holomorphy which proceeds like this see Theorem 5.4 .5 of $[12, \mathrm{p} .128]$, which is basically the analog of Theorem 1.5 (in $[12,5.4 .5], O(\Omega)$ separates points and all functions holomorphic in $\Omega$ are simultaneously analytically continued by forming power series expansions).

LEMMA 2.18. Let $\Omega$ be a Riemann domain and let $O(\Omega)$ be preordered by $<($ where $<$ is defined in 2.2). Then there exists a function $h \in O(\Omega)$ such that $f<h$ for all $f \in O(\Omega)$.

Proof. Let $h$ be the function of Lemma 2.17 and let $\left(\psi_{h}^{\prime}\right)^{-1}$ be the inverse of the biholomorphism $\psi_{h}$. Then the diagram

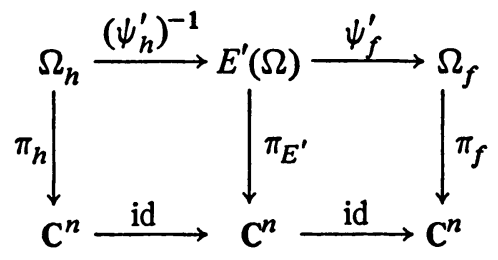


is commutative for every $f \in O(\Omega)$. Let $\psi_{h f}=\psi_{f}^{\prime} \circ\left(\psi_{h}^{\prime}\right)^{-1}$. Then the diagram above and the diagram preceding Lemma 2.10 show that the local biholomorphism $\psi_{h f}$ is a connecting map in the sense of Definition 2.2. Therefore $f<h$ for all $f \in O(\Omega)$, which proves the lemma.

THEOREM 2.19. Let $\Omega$ be a Riemann domain. Then $\widetilde{\Omega}$, the projective limit space of the projective spectrum $\left\{\Omega_{f} ; \psi_{f g}\right\}$, can be given the structure of a Riemann domain so that $\widetilde{\Omega}$ is an envelope of holomorphy for $\Omega$.

Proof. Let $h$ be the function of Lemma 2.17. Then Lemma 2.18 shows that $O(\Omega)$ is a directed set with $<$ and that $\{h\}$ is cofinal in $O(\Omega)$. By 2.7 of $\left[8\right.$, p. 431] the map $\mu_{h}: \widetilde{\Omega} \rightarrow \Omega_{h}$ is a homeomorphism (where $\mu_{h}$ is the restriction of $p_{h}: \Pi_{f} \Omega_{f} \rightarrow \Omega_{h}$ to $\left.\widetilde{\Omega}\right)$. For any $z \in \widetilde{\Omega}$ let $\mu_{f}(z)=z_{f}$. Then $\pi_{f}\left(z_{f}\right)=$ $\pi_{h}\left(z_{h}\right)$ for all $f \in O(\Omega)$. By 2.5 of $\left[8\right.$, p. 430] there exists a map $\tilde{\pi}: \widetilde{\Omega} \rightarrow \mathbf{C}^{n}$ such that the diagram

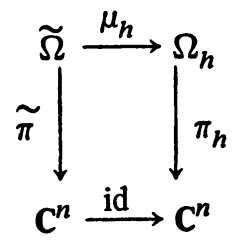

is commutative. Since $\mu_{h}$ is a homeomorphism, it follows that $\tilde{\pi}$ is a local homeomorphism. If we give $\widetilde{\Omega}$ the complex structure induced by $\tilde{\pi}$, then $\mu_{h}$ is a biholomorphism since the topology in $\widetilde{\Omega}$ is compatible with the complex structure determined by $\tilde{\pi}$. Thus $\widetilde{\Omega}$ is an envelope of holomorphy for $\Omega$. This completes the proof.

Since $\widetilde{\Omega}$ and $E^{\prime}(\Omega)$ are both biholomorphic to $\Omega_{h}$ we have that $I: \widetilde{\Omega} \stackrel{\text { id }}{\longrightarrow}$ $S(\Omega)$ is a biholomorphism, which gives

COROLlary 2.20. Let $\Omega$ be a Riemann domain. Then $\widetilde{\Omega}=S(\Omega)=$ $E^{\prime}(\Omega)$.

REMARKS. $E^{\prime}(\Omega)$ corresponds to the notion of "durchschnitt" of the family $\left\{\Omega_{f}\right\}$ given in [1, p. 11] (compare [6, p. 622]). It can occur, when $O(\Omega)$ does not separate points, that $\Omega$ is nonschlicht, but $\widetilde{\Omega} \subset \mathbf{C}^{n}$ (see [3, p. 177]). An example where $\Omega \subset \mathbf{C}^{n}$ and a nonschlicht $\widetilde{\Omega}$ is explicitly represented is given in [5, p. 428]. This phenomenon shows why in Definition 2.2 we cannot require that $\psi_{f g}$ be injective.

The constructions in [7] and [15] of an envelope of holomorphy for an arbitrary Riemann domain could be used to give a constructive definition of envelope of holomorphy. Here we shall use Theorem 2.19 to make the following 
DEFINITION 2.21. Let $\Omega$ be a Riemann domain. Then the projective limit space, $\widetilde{\Omega}$, of the projective spectrum $\left\{\Omega_{f}: \psi_{f g}\right\}$ (where $O(\Omega)$ is preordered by $<$ as in Definition 2.2) is an envelope of holomorphy for $\Omega$. Any complex manifold which is biholomorphic to $\widetilde{\Omega}$ is also an envelope of holomorphy for $\Omega$.

REMARK. Note that the uniqueness up to biholomorphism of envelopes of holomorphy of the same Riemann domain, first proved in [7], is an easy consequence of Lemma 2.18 .

III. Applications. The projective limit space representation of the envelope of holomorphy for a Riemann domain immediately gives the following theorems.

THEOREM 3.1. If $\pi(\Omega)$ is bounded, then $\tilde{\pi}(\widetilde{\Omega})$ is bounded; in particular, if $\pi_{f}\left(\Omega_{f}\right)$ is bounded for some $f \in O(\Omega)$, then $\tilde{\pi}(\widetilde{\Omega})$ is bounded.

Proof. If $\pi(\Omega)$ is bounded, then there is a function $g$ defined in a ball $B \supset \pi(\Omega)$, such that $B_{g}=B$. Then $g \circ \pi$ will be holomorphic on $\Omega$ with $\Omega_{g^{\circ} \pi}=$ B. Since $\tilde{\pi}(\widetilde{\Omega})=\bigcap_{f} \pi_{f}\left(\Omega_{f}\right)$, the result follows.

THEOREM 3.2. If $\tilde{\pi}$ is injective, $\left({ }^{1}\right)$ then $\bigcap_{f} \pi_{f}\left(\Omega_{f}\right)$ is an envelope of holomorphy for $\Omega$.

Proof. If $\tilde{\pi}$ is injective, then it is a biholomorphism. Thus, since $\widetilde{\Omega}$ is an envelope of holomorphy for $\Omega$, the image of $\widetilde{\Omega}$ under $\tilde{\pi}, \bigcap_{f} \pi_{f}\left(\Omega_{f}\right)$, is also an envelope of holomorphy for $\Omega$.

A geometric characterization of those domains in $\mathbf{C}^{n}$ possessing schlicht envelopes of holomorphy is still not known. However, a sufficient condition for $\widetilde{\Omega}$ to be schlicht is that $\tilde{\pi}(\widetilde{\Omega})$ be simply connected.

We now consider the notion of envelope of holomorphy of any connected subset of a Riemann domain $\Omega$, which was introduced in [11] for arbitrary subsets of a Stein manifold.

Let $S$ be a connected subset of the Riemann domain $\Omega$ where $O(\Omega)$ separates points, and let $\left\{U_{\alpha}: \alpha \in I\right\}$ be a fundamental system of open connected neighborhoods of $S$, which is assured by the local connectivity of $\Omega$. We preorder $I$ by $\alpha>\beta$ if and only if $U_{\alpha} \subset U_{\beta}$. In [11, p. 510] they show that if $\alpha>\beta$ then there exists a local biholomorphism $\psi_{\alpha \beta}: \widetilde{U}_{\alpha} \rightarrow \widetilde{U}_{\beta}$ such that $\widetilde{\pi}_{\beta} \circ \psi_{\alpha \beta}=\tilde{\pi}_{\alpha}$ where $\widetilde{U}_{\alpha}$ and $\widetilde{U}_{\beta}$ are the envelopes of holomorphy, in the sense of Rossi [15], for $U_{\alpha}$ and $U_{\beta}$ respectively. In [11] the envelope of holomorphy of $S, \widetilde{S}$, is defined to be the spectrum of the algebra $O(S)(\widetilde{S}$ is the set of nonzero continuous homomorphisms of the algebra $O(S)$ into $\mathbf{C}$, where $O(S)=\left\{f \mid S: f \in O\left(U_{\alpha}\right)\right.$ for some $\alpha \in$ $\eta$ is a direct limit space). Then $\widetilde{S}$ is characterized as the projective limit space of

(1)When $\tilde{\pi}$ is injective, $\widetilde{\Omega}$ is said to be schlicht and $\tilde{\pi}(\widetilde{\Omega})$ is often called the schlicht envelope of holomorphy of $\Omega$. 
the projective spectrum $\left\{\widetilde{U}_{\alpha}: \psi_{\alpha \beta}\right\}$. Let $g: S \rightarrow \widetilde{S}$ be point evaluation. The set $S$ is said to be holomorphically convex if $g(S)=\widetilde{S}$ and if $g$ is injective. Using this definition, Cartan's Theorems A and B are proved in [11] for coherent analytic sheaves on a compact holomorphically convex subset of a Stein manifold. The projective limit space representation is crucial in doing this. For this and other results which depend on this representation see [11] and [18].

We shall now show that the envelope of holomorphy of an arbitrary connected subset $S$ of a Riemann domain as defined in [11] is homeomorphic to the projective limit space of "Riemann surfaces" of functions in $O(S)$, the analog of Definition 2.21 for arbitrary sets. Using this fact we then give a characterization of connected holomorphically convex subsets of a holomorphically convex Riemann domain $\Omega$ (it should be pointed out that in [11] $\Omega$ need not be a Riemann domain).

Let $\Omega$ be a holomorphically convex Riemann domain and let $S$ be any connected subset of $\Omega$. We preorder $O(S)$ as follows: $g<f$ if and only if (1) there exist domains $U_{\alpha}$ and $U_{\beta}$, where $S \subset U_{\alpha} \subset U_{\beta}, f \in O\left(U_{\alpha}\right)$ and $g \in O\left(U_{\beta}\right)$, and if (2) there exists a local biholomorphism $\psi_{f g}:\left(U_{\alpha}\right)_{f} \rightarrow\left(U_{\beta}\right)_{g}\left(\left(U_{\alpha}\right)_{f}\right.$ and $\left(U_{\beta}\right)_{g}$ as in Theorem 1.5) such that $\pi_{g}{ }^{\circ} \psi_{f g}=\pi_{f}$, and $\chi_{g}=\psi_{f g}{ }^{\circ} \chi_{f}$ on $U_{\alpha} \cap U_{\beta}$. Since $I$ is a directed set and can be identified with a subset of $O(S)$, we have from 2.7 of $[8$, p. 431] the following

THEOREM 3.3. Let $\Omega$ be a holomorphically convex Riemann domain and let $S$ be any connected subset of $\Omega$. Then the projective limit spaces of the projective spectrums $\left\{\widetilde{U}_{\alpha} ; \psi_{\alpha \beta}\right\}$ and $\left\{\left(U_{\alpha}\right)_{f} ; \psi_{f g}\right\}$, where $\alpha \in I$ and $f \in O(S)$, are homeomorphic.

Let $\hat{S}=\lim \operatorname{proj}_{f \in O(S)}\left(U_{\alpha}\right)_{f}$ and define $\chi: S \rightarrow \hat{S}$ by $\chi(s)=\left\{\chi_{f}(s): f \in\right.$ $O(S)\} \in \hat{S}$ for $s \in S$. Let $\mu_{f}: \hat{S} \rightarrow\left(U_{\alpha}\right)_{f}$ be the restriction to $\hat{S}$ of projection onto the fth factor.

Just as in Theorem 3.3 we can obtain a homomorphism $\gamma: \widetilde{S} \rightarrow \hat{S}$ such that $\gamma \circ g=\chi$. Thus each $f \in O(S)$ lifts to $\tilde{S}$ since for every $f$ there exists $\tilde{f}$ satisfying

in $S$.

$$
f=\tilde{f} \circ \chi_{f}=\tilde{f} \circ \mu_{f} \circ \chi=f \circ \mu_{f} \circ \gamma \circ g
$$

Let $\pi_{\widetilde{S}}: \widetilde{S} \rightarrow \Omega$ be the map $\pi$ of 2.2 in $[11$, p. 510$]$ ( $\pi_{\widetilde{S}}$ is the composition of the natural map from $\widetilde{S}$ into $\widetilde{\Omega}$, the spectrum of $O(\Omega)$, with a biholomorphism from $\widetilde{\Omega}$ onto $\Omega$ ).

THEOREM 3.4. Let $\Omega$ be a holomorphically convex Riemann domain and let $S$ be any subset of $\Omega$ such that $\widetilde{S}$ is connected and $\pi_{\mathfrak{S}}(\widetilde{S})=S$. Then $S$ is holomorphically convex in the sense of [11], i.e., $g: S \rightarrow \widetilde{S}$ is injective and surjective. 
Proof. Since $\Omega$ is holomorphically convex, $O(\Omega)$ separates points in $\Omega$; hence $O(S)$ separates points in $S$. This implies that $g$ is injective.

Let $z$ be any point in $\widetilde{S}$. Take any point $w \in g(S)$. For each $f \in O(S)$, the value of the extension of $f$ to $\left(U_{\alpha}\right)_{f}$ at $\mu_{f} \circ \gamma(z)$ is obtained by analytic continuation of $f$ in a neighborhood of a connected set in $\Omega$ containing $\pi_{\mathcal{S}}(z)$ and $\pi_{\widetilde{S}}(w)$. From the connectivity of $\widetilde{S}$ and the fact that $\pi_{\mathcal{S}}(\widetilde{S})=S$, it follows that $S$ is such a connected set which works for every $f$. Then it must be that $w \in g(S)$ since $O(S)$ separates points in $S$. The proof is complete.

REMARKS. (1) The notion of envelope of holomorphy of a Riemann domain is a formal analogue of the notion of convex hull of a domain in $\mathbf{R}^{n}$ in the following sense.

The convex hull of a domain is the intersection of all convex domains containing the given domain. This can be characterized as the projective limit space of these convex domains where the connecting maps are identity maps. Furthermore, by 1.5 of $\left[5\right.$, p. 417] for each convex domain there exists a $C^{\infty}$ convex function which goes to infinity at each boundary point. Thus the convex hull of a domain in $\mathbf{R}^{n}$ can be realized as the projective limit space of a family of "domains of convexity" for a certain class of convex functions in the given domain.

(2) For an example of a convex function in a domain in $\mathbf{R}^{n}$ which does not extend to a convex function on the convex hull of the domain see $[4, \mathrm{p} .80]$. The example in [5, p. 428] of a domain in $\mathbf{C}^{2}$ whose envelope of holomorphy is not spread over a domain of holomorphy shows that the intersection of all domains of holomorphy containing a given domain may not even be a domain of holomorphic continuation for all holomorphic functions in the given domain.

(3) Explicit formulas for the envelopes of holomorphy for certain connected sets (Reinhardt sets which need not contain the origin, certain tube sets, and complete Hartogs sets in $\mathbf{C}^{2}$ ) are obtained in [5].

\section{BIBLIOGRAPHY}

1. H. Behnke and P. Thullen, Theorie der Funktionen mehrerer komplexer Veränderlichen, Ergebnisse der Mathematik und ihrer Grenzgebiete, Band 51, Zwiete, erweiterte Auflage, Springer-Verlag, Berlin and New York, 1970. MR 42 \#6274.

2. E. Bishop, Holomorphic completions, analytic continuation, and the interpolation of semi-norms, Ann. of Math. (2) 78 (1963), 468-500. MR 27 \#4958.

3. H.-J. Bremermann, Construction of the envelopes of holomorphy of arbitrary domains, Rev. Mat. Hisp.-Amer. (4) 17 (1957), 175-200. MR 19, 880.

4. - On the conjecture of the equivalence of the plurisubharmonic functions and the Hartogs functions, Math. Ann. 131 (1956), 76-86. MR 17, 1070.

5. R. Carmignani, Envelopes of holomorphy and holomorphic convexity, Trans. Amer. Math. Soc. 179 (1973), 415-431. MR 47 \#5296.

6. H. Cartan und P. Thullen, Zur theorie der Singularitäten der Funktionen mehrer Veränderlichen. Regularitäts-und Konvergenzbereiche, Math. Ann. 106 (1932), 617-647.

7. Séminaires $H$. Cartan, École Normale Supérieure, 1951/52, Secrétariat mathématique, Paris, 1953. MR 16, 233. 
8. J. Dugundji, Topology, Allyn and Bacon, Boston, Mass., 1966. MR 33 \#1824.

9. R. Gunning and H. Rossi, Analytic functions of several complex variables, PrenticeHall Ser. in Modern Analysis, Prentice-Hall, Englewood Cliffs, N. J., 1965. MR 31 \#4927.

10. R. Hartogs, Über die aus den singulären Stellen einer analytischen Funktion mehrerer Veränderlichen bestchenden Gebilde, Acta. Math. 32 (1909), 57-79.

11. R. Harvey and R. O. Wells, Jr., Compact holomorphically convex subsets of a Stein manifold, Trans. Amer. Math. Soc. 136 (1969), 509-516. MR 38 \#3470.

12. L. Hörmander, An introduction to complex analysis in several variables, Van Nostrand, Princeton, N. J., 1966. MR 34 \$2933.

13. B. Malgrange, Lectures on the theory of functions of complex variables, Tata Institute of Fundamental Research, Bombay, 1958.

14. K. Oka, Sur les fonctions analytiques de plusieurs variables. IX. Domaines finis sans point critique intérieur, Japan J. Math. 23 (1953), 97-155 (1954). MR 17, 82.

15. H. Rossi, On envelopes of holomorphy, Comm. Pure Appl. Math. 16 (1963), 9-17. MR 26 \#6436.

16. P. Thullen, Zur Theorie der Singuläritäten der Funktionen zweier komplexer Veränderlichen. Die Regularitätshüllen, Math. Ann. 106 (1932), 64-76.

17. V. S. Vladimirov, Methods of the theory of functions of several complex variables, "Nauka", Moscow, 1964; English transl., M. I. T. Press, Cambridge, Mass., 1966. MR 30 \#2163; 34 \#1551.

18. R. O. Wells, Jr., Function theory on differentiable submanifolds, Contribution to Analysis-A Collection of Papers Dedicated To Lipman Bers, Academic Press, New York and London, 1974, pp. 407-441.

DEPARTMENT OF MATHEMATICS, UNIVERSITY OF MISSOURI, COLUMBIA MISSOURI 65201 